\title{
Remotely activated, vibrational magnetoelastic array system for controlling cell adhesion
}

\author{
Steven Trierweiler, Hallie Holmes, Brandon Pereles, Rupak Rajachar, Keat Ghee Ong* \\ Department of Biomedical Engineering, Michigan Technological University, Houghton, USA \\ Email: *kgong@mtu.edu
}

Received 21 February 2013; revised 24 March 2013; accepted 1 April 2013

Copyright (c) 2013 Steven Trierweiler et al. This is an open access article distributed under the Creative Commons Attribution License, which permits unrestricted use, distribution, and reproduction in any medium, provided the original work is properly cited.

\begin{abstract}
A new system was designed to selectively control cellular adhesion to medical implants. The system is based on magnetoelastic (ME) materials that can be remotely set to generate mechanical vibrations at submicron levels with predetermined amplitude and frequency. Previous studies have demonstrated the capacity of these vibrations to control cellular adhesion at a substrate surface. In this work, an ME film with two conjoined strips was developed to investigate the potential of this system to provide region specific control of cellular adhesion. In vitro cell culture experiments performed with L929 fibroblasts indicate that cellular adhesion can be increased or decreased at different regions of the film by changing the frequency of the magnetic field.
\end{abstract}

Keywords: Cellular Adhesion; Vibration; Magnetoelastic Materials

\section{INTRODUCTION}

The host response plays a significant role in the longevity and success of implantable devices. For example, fibrosis, defined as excessive growth of fibrous connective tissues during a reparative or reactive process, may lead to reduction in the functional stability of an implant as the overgrown tissues can often lead to tissue consolidation and increased stiffness local to the implant, eventually significantly affecting performance [1]. Post-implantation host response often starts with an acute regenerative phase followed by a chronic phase [1]. The intensity and outcomes of these reactions are dependent on the physical and chemical properties of the implantable device, as well as the local environment [2]. As a result, a number of strategies have been developed to minimize

"Corresponding author. the host response by altering the surface properties (surface roughness, hydrophobicity, stiffness, etc.) [3-6] of implants or controlling the local environment [7].

In previous work, we established a possible therapeutic technique for controlling pathological fibrosis. The technique is based on magnetoelastic (ME) materials that can be remotely set to generate a static or dynamic strain of predetermined amplitude and frequency when an AC magnetic field is applied [8]. When activated by the magnetic field, the ME material changes dimensions, effectively converting magnetic energy to mechanical energy in the form of vibrations [8]. The advantage of this technique is that these materials can be remotely activated post-implantation to modulate and monitor cell adhesion.

Previous results indicated that although the ME material was not biocompatible, its biocompatibility could be significantly improved with proper surface functionalization [8-10]. For example, in vivo and in vitro experiments indicated that Parylene-C coatings provide longterm stability to the ME material [9]. Previous experiments also indicated that submicron vibrations $(0.1 \mu \mathrm{m}$ $0.15 \mu \mathrm{m}$ ) cause a significant decrease in adhesion of fibroblasts [8-10]. Furthermore, the vibrations did not induce apoptosis [8], indicating that this approach may be a viable solution to control fibrosis.

The primary goal of this work is to create a surface that would allow for spatial control of local cell adhesion. In addition, this work investigates whether very low amplitude vibrations $(<10 \mathrm{~nm})$ can increase fibroblast adhesion even though larger vibrations $(0.1-0.15 \mu \mathrm{m})$ have been shown to decrease adhesion. To achieve these goals, two tasks were performed: 1) a single ME strip was used to test if very low vibrations $(<10 \mathrm{~nm})$ can increase fibroblast adhesion, and 2) an ME film with two conjoined strips was used to investigate the ability of ME vibrations to provide unique levels of cellular adhesion on each strip, where regional vibrations can be controlled independently by altering the frequency of the magnetic field. 


\section{METHODS AND MATERIALS}

\subsection{Fabrication of the ME Film}

A commercial magnetoelastic material, Metglas 2826MB $\left(\mathrm{Fe}_{40} \mathrm{Ni}_{38} \mathrm{Mo}_{4} \mathrm{~B}_{18}\right)$ from Metglas, Inc., was used to create ME films for both experiments. The material was a 12.7 mm wide ribbon with a thickness of $28 \mu \mathrm{m}$. For the first task, the ME films were rectangles created by mechanically shearing the Metglas ribbon into strips with dimensions of $12.7 \mathrm{~mm} \times 5 \mathrm{~mm}$. The strips were sonicated in isopropanol for 5 minutes and annealed for 2 hours at $125^{\circ} \mathrm{C}$ to remove internal stress. For the second task, two rectangular conjoined strips of $12.3 \mathrm{~mm} \times 2.7 \mathrm{~mm}$ and $13.7 \mathrm{~mm} \times 2.7 \mathrm{~mm}$ were created. The strips were separated by a gap of $1.0 \mathrm{~mm}$ via a bridge of $0.5 \mathrm{~mm}$ wide (see Figure 1). The conjoined ME strips were created with an etching process. First, both sides of the as-purchased ME ribbon were coated with Nazdar $^{\circledR} 59,000$ Series Enamel Plus Gloss Screen Ink 59,192. After the ink dried, the outline of the conjoined strips was carved on one of the painted surface with a CNC Microplotter Mill, exposing the ME material beneath. The patterned ME ribbon was then immersed in a ferric chloride etching bath for $20-30$ minutes at $45^{\circ} \mathrm{C}$ to etch out of the conjoined strips. The ink was removed from the strips by rubbing with acetone and the conjoined strips were then sonicated and annealed as previously described. Parylene-C was coated onto the ME films for both experiments using a Parylene deposition system (PDS 2010 LABCOTER $^{\mathrm{TM}}$ 2) with the manufacturer's recommended protocol. The films were then etched with oxygen plasma (200 mTorr) using a March Jupiter II RIE system to create a surface conducive to cell adhesion. All ME films were sterilized with ethylene oxide gas before cell culture.

\subsection{Characterization of the ME Film}

The ME films were vibrated independently using a customized system previously developed [8-10]. The ME films were placed in a chamber slide within a magnetic coil. A custom, microcontroller-based ME interrogation

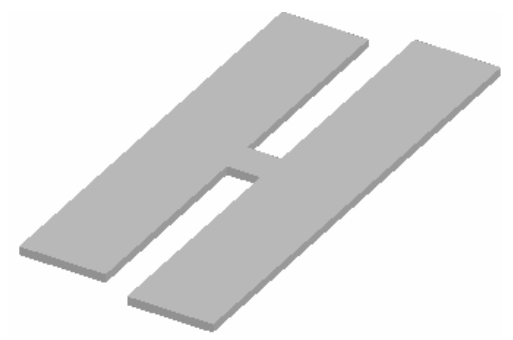

Figure 1. Illustration of the two conjoined ME strips, which have two distinct resonant frequencies so each strip can be set to vibrate independently. system was used to generate the excitation magnetic fields. The resonant behavior of the ME films were determined by measuring their magnetic field response at varying frequencies.

\subsection{Cell Culture Experiments}

L929 fibroblasts (ATCC CCCL-1) were used in all experiments involving cell culture. The cells were maintained in standard culture medium composed of $10 \%$ fetal bovine serum (FBS) and 0.5\% ATCC Penicillin/ Streptomycin in Dulbecco's Modified Eagles Medium (Cellgro Catalog No. 10-017-CM). Chamber slides (LabTek $^{\mathrm{TM}}$-Chamber Slide ${ }^{\mathrm{TM}}$ system) were used to hold the ME films and cell cultures. Calcien-AM (Fluka 11783) was used to fluorescently label live cells adhered to the ME substrate surface. Images were taken with an upright Olympus BX51 microscope, and cellular adhesion was determined by direct cell counting.

The rectangular ME films used in the first task were placed in the chamber slide, followed by cells seeded at a density of $1 \times 10^{4}$ cells $/ \mathrm{cm}^{2}$. These ME films were separated into two groups: control (no vibrational loading), and vibrational loading at $171-176 \mathrm{kHz}$ which was at least $10 \mathrm{kHz}$ higher than the resonant frequency of the strip. After 48 hours of incubation at $37^{\circ} \mathrm{C}$ and $5 \% \mathrm{CO}_{2}$, $\mathrm{ME}$ vibrations were applied to the ME film for 1 hour followed by staining with Calcien-AM.

For the second task, the two conjoined ME strips were separated into three groups: control (no vibrational loading); vibrational loading at $171-176 \mathrm{kHz}$, and vibrational loading at 152 - $157 \mathrm{kHz}$. The experimental process was identical to that previously described for the first task.

\subsection{Statistical Analysis}

Statistical analyses were made using standard student's t-test or analysis of variance (ANOVA) on JMP software. All experiments were conducted in triplicate, unless otherwise stated. Data is expressed as the mean \pm S.E.M. (standard error of the mean) and $p$-values less than 0.05 $(p<0.05)$ were considered significant.

\section{RESULTS AND DISCUSSION}

\subsection{Characterization of the ME Film}

The resonance of the single strip ME film is plotted in Figure 2. The resonant frequency of the strip was about $160 \mathrm{kHz}$. Figure 2 also indicates the frequency range of excitation field applied to the single strip ME films, which was at least $10 \mathrm{kHz}$ higher than the resonant frequency of these strips. The resonance of the two-strip ME film is shown in Figure 3. As each ME strip has a distinct resonant frequency, two resonant peaks were ob- 


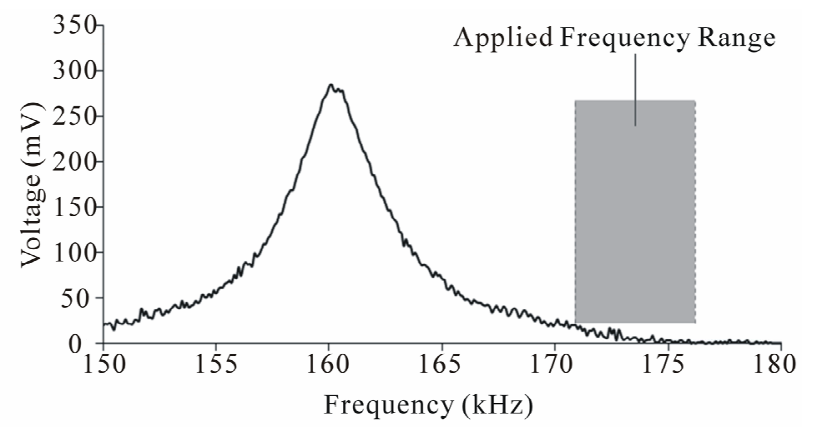

Figure 2. The resonance of the single rectangular ME strip. The excitation frequency range is also indicated.

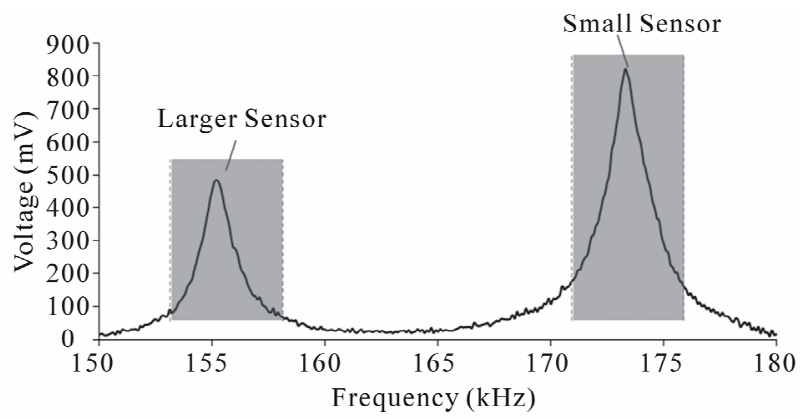

Figure 3. The resonances of the two conjoined ME strips. The excitation frequency ranges are also indicated. Two sets of vibrational loads were applied: one occurred at a frequency that included the resonant frequency of the larger strip while the other for the smaller strip.

served. The excitation frequencies applied to these films are also indicated.

\subsection{Cell Culture on ME Films}

Adhesion of L929 fibroblasts to the ME films can be controlled by changing the vibration magnitude, which can be easily achieved by changing the frequency of the excitation field (see Figure 3). As shown in Figure 4 A)-F), qualitative images of fibroblast adhesion indicated significant differences between controls and vibrated groups (at resonant frequency and off-resonant frequency) when quantified. Compared to the controls (no vibration), ME strips that were vibrated at the resonant frequency showed a significant decrease. On the other hand, ME strips that were vibrated at off resonant frequency showed an increase in cell adhesion when compared to the controls.

As indicated in Figure 5, ME films that were vibrated at the resonant frequency showed significant reduction in cell adhesion $(p<0.05)$ while films that were vibrated at frequencies away from the resonance showed an increase in cell adhesion. These quantitative results demonstrate that the larger vibrations $(0.1-0.15 \mu \mathrm{m})$ inhibited cell adhesion. However, at low vibration levels $(<10 \mathrm{~nm})$, a result of excitation by magnetic fields of frequencies at least $10 \mathrm{kHz}$ from the resonant frequency, cell adhesion increased. Therefore, these results suggest that ME materials have the potential to create a surface that can be precisely tuned for increasing or decreasing cell adhesion for specific applications.

Results from Figures $\mathbf{4}$ and $\mathbf{5}$ indicate that under certain conditions, vibrations can also increase cell adhesion. This behavior was not reported in our previous work that focused on vibrational strains at $0.1-0.15 \mu \mathrm{m}$. To ensure the increase in cell adhesion is not caused by the interference from the neighboring vibrating strip, single strip ME films (task 1) were used to load cells with off frequency vibrations. Similar to the observations on 2-stip ME films, qualitative results from the single-strip ME films indicated that application of very small vibrations at off resonant frequency increased fibroblast adhesion compared to static controls. This observation is consistent with other studies, in which nanometer vibrations also showed an increase in fibroblast adhesion [11]. Quantitative results indicated that off-resonant frequency vibrations significantly increase $(p<0.05)$ fibroblast
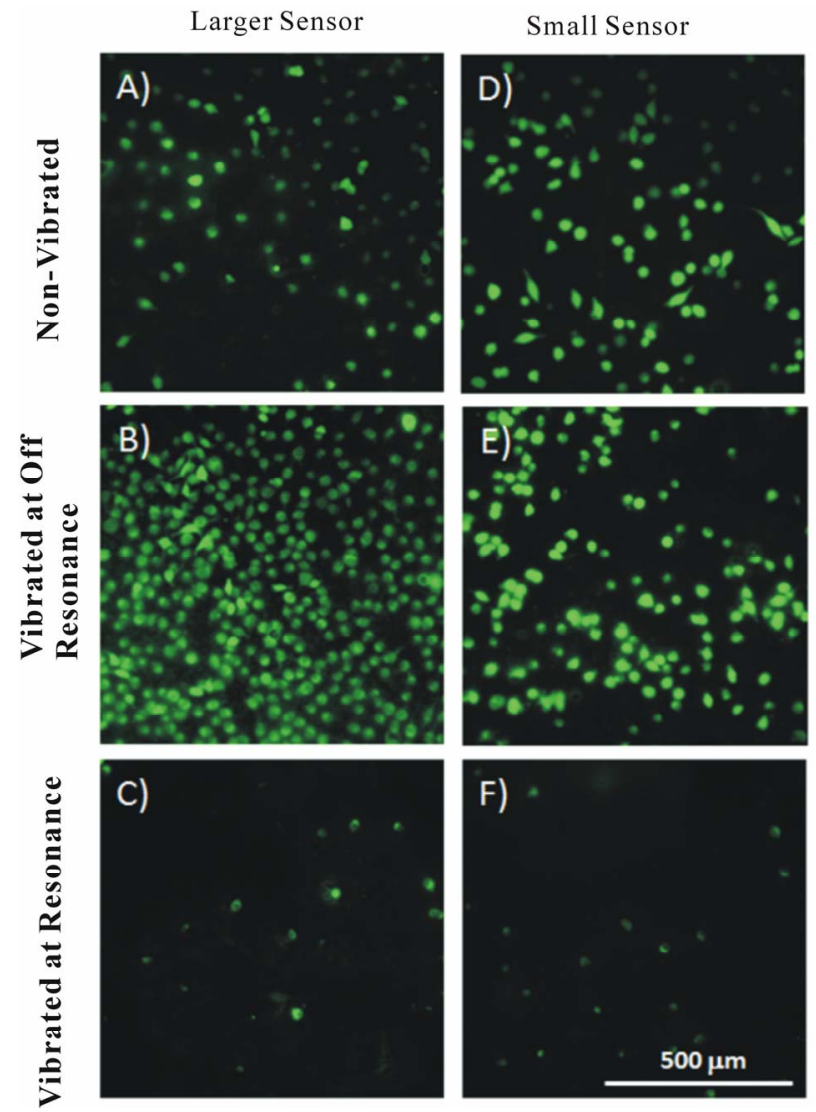

Figure 4. Qualitative results showing the effects of ME vibrations on fibroblast cell adhesion on plasma-treated Parylene-C coated ME films. Images of fibroblast cells show differences in adhesion on the large strip A)-C) and small strip D)-F) between non-vibrated, vibrated at off-resonant frequency, and vibrated at resonant frequency. 


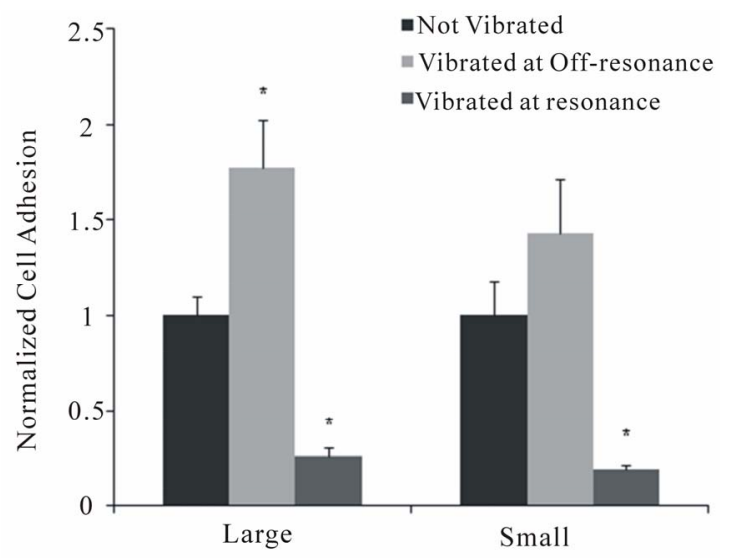

Figure 5. Effects of ME vibrations on fibroblast cell adhesion to plasma-treated Parylene-C coated ME films. ME applied vibrations greatly affect fibroblast cell adhesion. Larger vibrations were shown to significantly decrease cell adhesion while smaller ME vibrations were shown to increase cell adhesion. Statistically significant differences $(p<0.05)$ between groups are indicated $\left(^{*}\right)$.
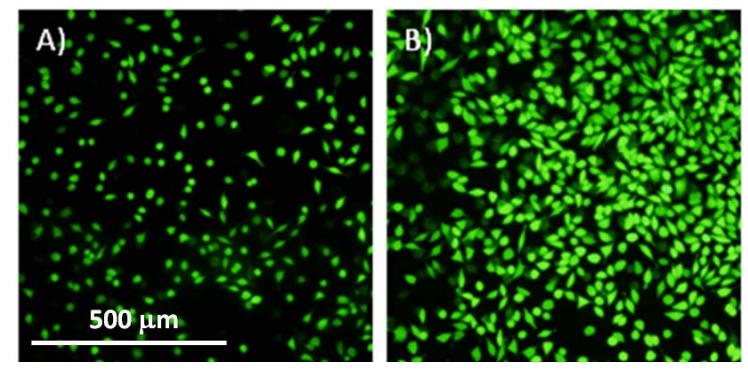

Figure 6. Qualitative results showing effects of ME vibrations on fibroblast cell adhesion to plasma-treated Parylene-C coated ME films. Images of fibroblast cells show differences in cell adhesion between non-vibrated A) and vibrated at off resonant frequency B).

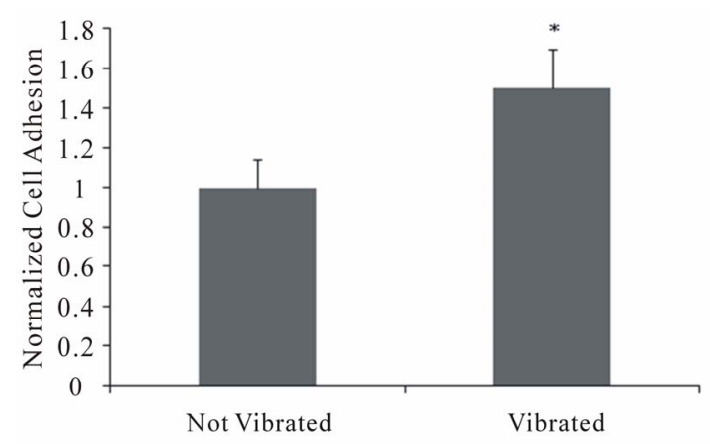

Figure 7. Effects of vibrations $(<10 \mathrm{~nm})$ on fibroblast adhesion to plasma-treated Parylene-C coated ME films. Small vibrations were shown to significantly increase cell adhesion. Statistically significant differences $(p<0.05)$ between groups are indicated $\left(^{*}\right)$.

adhesion to ME materials, as illustrated in Figures 6 and 7. These results demonstrate that ME materials can be designed to increase or decrease fibroblast cell adhesion depending on desired cell attachment response.

\section{CONCLUSION}

In vitro experiments showed that vibrational ME films can be used to control cell adhesion by changing the dimension of the material and/or the excitation frequency. By applying an AC magnetic field at the resonance of the ME film (vibration strain of $0.1-0.15 \mu \mathrm{m}$ ) local adhesion of fibroblasts was decreased. However, by applying an AC magnetic field at a frequency far from the ME film's resonant frequency (vibration strain of $<10 \mathrm{~nm}$ ), an increase in local fibroblast adhesion was observed. The ability to increase or decrease cell adhesion may allow for the design of a complex implant interface where local cell adhesion behavior can be tailored in concert with surface modification to improve overall long-term implant integration and stability.

\section{ACKNOWLEDGEMENTS}

Research reported in this publication was partially supported by $\mathrm{Na}$ tional Institute of Biomedical Imaging and Bioengineering of the National Institutes of Health under award number NIH1R03EB01450401A1. The content is solely the responsibility of the authors and does not necessarily represent the official views of the National Institutes of Health.

\section{REFERENCES}

[1] Wynn, T.A. (2008) Cellular and molecular mechanisms of fibrosis. The Journal of Pathology, 214, 199-210. doi:10.1002/path.2277

[2] Van Luyn, M.J.A., Harmsen, M. and Luttikhuizen, D. (2006) Cellular and molecular dynamics in the foreign body reaction. Tissue Engineering, 12, 1955-1970. doi:10.1089/ten.2006.12.1955

[3] Liu, X.M., Lim, J.Y., Donahue, H.J., Dhurjati, R., Mastro, A.M. and Vogler, E.A. (2007) Influence of substratum surface chemistry/energy and topography on the human fetal osteoblastic cell line hFOB 1.19: Phenotypic and genotypic responses observed in vitro. Biomaterials, 28, 4535-4550. doi:10.1016/j.biomaterials.2007.06.016

[4] Khang, G., Lee, S.J., Lee, J.H., Kim, Y.S. and Lee, H.B. (1999) Interaction of fibroblast cells on poly(lactide-coglycolide) surface with wettability chemogradient. BioMedical Materials and Engineering, 9, 179-187.

[5] Webb, K., Hlady, V. and Tresco, P.A. (1998) Relative importance of surface wettability and charged functional groups on NIH 3T3 fibroblast attachment, spreading, and cytoskeletal organization. Journal of Biomedical Materials Research, 41, 422-430.

[6] Chen, C.J., Tan, J. and Tien, J. (2004) Mechanotransduction at cell-matrix and cell-cell contacts. Annual Review of Biomedical Engineering, 6, 275-302. doi:10.1146/annurev.bioeng.6.040803.140040

[7] Wilson, C.J., Clegg, R.E., Leavesley, D.I. and Pearcy, 
M.J. (2005) Mediation of biomaterial-cell interactions by adsorbed proteins: A review. Tissue Engineering, 11, 118. doi:10.1089/ten.2005.11.1

[8] Vlaisavljevich, E., Janka, L.P., Ong, K.G. and Rajachar, R.M. (2011) Magnetoelastic materials as novel bioactive coatings for the control of cell adhesion. IEEE Transactions on Biomedical Engineering, 58, 698-704. doi:10.1109/TBME.2010.2093131

[9] Holmes, H., Tan, E.L., Ong, K.G. and Rajachar, R.M. (2011) Real-time, in vivo investigation of mechanical stimulus on cells with remotely activated, vibrational mag- netoelastic layers. IEEE Engineering in Medicine and Biology Society Annual International Conference, 39793982.

[10] Holmes, H.R., Tan, E.L., Ong, K.G. and Rajachar, R.M. (2012) Fabrication of biocompatible. Vibrational magnetoelastic materials for controlling cellular adhesion. Biosensors, 2, 57-69. doi:10.3390/bios2010057

[11] Ito, Y., Kimura, T., Ago, Y., Nam, K., Hiraku, K., Miyazaki, K., Masuzawa, T. and Kishida, A. (2011) Nano-vibration effect on cell adhesion and its shape. Bio-Medical Materials and Engineering, 21, 149-158. 